\title{
Correction to: The assessment of severe lexical disorders in Italian individuals with aphasia
}

\author{
Laura Veronelli ${ }^{1}$. Ilaria Scola ${ }^{2}$ - Mirella Frustaci ${ }^{3} \cdot$ Massimo Corbo $^{1} \cdot$ Claudio Luzzatti $^{4,5}$
}

Published online: 3 April 2020

(C) Fondazione Società Italiana di Neurologia 2020

\section{Correction to: Neurological Sciences (2020). https://doi.org/10.1007/s10072-020-04262-3}

This article was published with incomplete Table 4. The Equivalent scores were missing during the submission. The correct Table is presented here.
Publisher's note Springer Nature remains neutral with regard to jurisdictional claims in published maps and institutional affiliations.

Table 4 Age- and education-adjusted grids and equivalent scores for the confrontation naming task

\begin{tabular}{|c|c|c|c|c|c|c|c|c|c|c|c|c|c|c|}
\hline \multirow[b]{2}{*}{ Education } & \multicolumn{14}{|l|}{ Age } \\
\hline & 25 & 30 & 35 & 40 & 45 & 50 & 55 & 60 & 65 & 70 & 75 & 80 & 85 & 90 \\
\hline 5 & 1.13 & 1.25 & 1.38 & 1.53 & 1.68 & 1.85 & 2.04 & 2.24 & 2.48 & 2.75 & 3.08 & 3.47 & 3.98 & 4.70 \\
\hline 8 & -0.41 & -0.29 & -0.16 & -0.01 & 0.14 & 0.31 & 0.50 & 0.71 & 0.94 & 1.22 & 1.54 & 1.93 & 2.45 & 3.16 \\
\hline 10 & -0.92 & -0.80 & -0.67 & -0.53 & -0.37 & 0.31 & -0.02 & 0.19 & 0.43 & 0.70 & 1.03 & 1.42 & 1.93 & 2.65 \\
\hline 13 & -1.40 & -1.27 & -1.14 & -1.00 & -0.85 & -0.68 & -0.49 & -0.28 & -0.04 & 0.23 & 0.55 & 0.95 & 1.46 & 2.18 \\
\hline 17 & -1.77 & -1.65 & -1.51 & -1.37 & -1.22 & -1.05 & -0.86 & -0.65 & -0.42 & -0.14 & 0.18 & 0.58 & 1.09 & 1.81 \\
\hline \multicolumn{15}{|c|}{ Equivalent scores } \\
\hline \multicolumn{3}{|l|}{0} & \multicolumn{3}{|l|}{1} & \multicolumn{3}{|l|}{2} & \multicolumn{3}{|l|}{3} & \multicolumn{3}{|l|}{4} \\
\hline \multicolumn{3}{|l|}{$\leq 52.08$} & \multicolumn{3}{|c|}{$52.09-61.12$} & \multicolumn{3}{|c|}{$61.13-62.25$} & \multicolumn{3}{|c|}{$62.26-62.58$} & \multicolumn{3}{|l|}{$\geq 62.59$} \\
\hline
\end{tabular}

$*$ Corrected score $=$ raw score $-[4.084 *(\log (100$-age $)-1.614)]+[20.519 *(1 /$ educ-0.093 $)]$

The formula contains the model that permits the calculation of the correction factor for individuals who do not fit the age/education combinations for which pre-calculated correction factors are provided.

The online version of the original article can be found at https://doi.org/10. 1007/s10072-020-04262-3

Laura Veronelli

l.veronelli@ccppdezza.it

1 Department of Neurorehabilitation Sciences, Casa di Cura del Policlinico, Milan, Italy

2 Istituti Clinici Scientifici Maugeri - IRCCS di Montescano, Pavia, Italy
Rho-Passirana Hospital, ASST Rhodense, Milan, Italy

4 Department of Psychology, University of Milano-Bicocca, Milan, Italy

5 Milan Centre for Neuroscience, Milan, Italy 